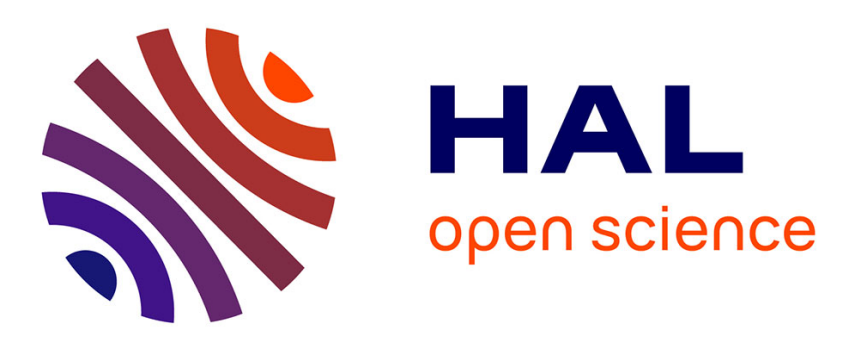

\title{
Dynamic vibration mode decomposition of auto-oscillating vocal fold replicas without and with vertical tilting
}

\author{
Annemie van Hirtum, Anne Bouvet, Isao Tokuda, Xavier Pelorson
}

\section{To cite this version:}

Annemie van Hirtum, Anne Bouvet, Isao Tokuda, Xavier Pelorson. Dynamic vibration mode decomposition of auto-oscillating vocal fold replicas without and with vertical tilting. Journal of Sound and Vibration, 2022, 516, 10.1016/j.jsv.2021.116504 . hal-03363196

\author{
HAL Id: hal-03363196 \\ https://hal.science/hal-03363196
}

Submitted on 3 Oct 2021

HAL is a multi-disciplinary open access archive for the deposit and dissemination of scientific research documents, whether they are published or not. The documents may come from teaching and research institutions in France or abroad, or from public or private research centers.
L'archive ouverte pluridisciplinaire HAL, est destinée au dépôt et à la diffusion de documents scientifiques de niveau recherche, publiés ou non, émanant des établissements d'enseignement et de recherche français ou étrangers, des laboratoires publics ou privés. 


\title{
Dynamic vibration mode decomposition of auto-oscillating vocal fold replicas without and with vertical tilting
}

\author{
Annemie Van Hirtum ${ }^{1}$, Anne Bouvet \\ LEGI, UMR CNRS 5519, Grenoble Alpes University, France \\ Isao Tokuda \\ Dep. Mech. Eng., Ritsumeikan Univ., Nojihigashi, Kusatsu, Shiga 525-8577, Japan \\ Xavier Pelorson \\ LEGI, UMR CNRS 5519, Grenoble Alpes University, France
}

\begin{abstract}
Vertical left-right level difference due to angular asymmetry characterises unilateral vocal fold paralysis. High-speed image sequences of three distinct autooscillating silicone vocal folds replicas are analysed simultaneously in both space and time with a dynamic vibration mode decomposition while imposing different degrees of angular asymmetry. From the modes eigenvalue spectra, it is found for all three replicas that the degree of angular asymmetry affects the decay of vibration modes. More in particular, for the assessed VF replicas, increased mode decay is observed when the replica contains a stiff epithelium-like surface layer whereas it decreases otherwise. Spatial mode patterns near the glottal aperture reflect the mode order along the posterior-anterior direction and the imposed angular asymmetry reduces the spatial mode extent near the tilted VF edge. Consequently, the quantified dynamic vibration mode properties, including the ones observed for higher order modes, are of potential interest for clinical studies involving high-speed vocal folds auto-oscillation imaging.
\end{abstract}

Keywords: High-speed vibration imaging, Dynamic vibration mode analysis,

\footnotetext{
${ }^{1}$ annemie.vanhirtum@univ-grenoble-alpes.fr
} 
Unilateral vocal fold paralysis, Mechanical vocal fold replicas

\section{Introduction}

Unilateral vocal fold paralysis (UVFP) is a common vocal fold (VF) pathology characterised by an air escape due to left-right VF asymmetries of the VF's shape, tension or/and positioning [1 [5]. The glottic insufficiency associated

5 with UVFP is reported to lead to dysphonia as air leakage is often associated with breathy voice or vocal fatigue [1, 6].

Each aspect of the fluid-structure (FS) interaction underlying normal or abnormal vocal fold vibration can be studied systematically in controlled and known conditions from experiments using deformable mechanical VF replicas 7 11. In the current work, three molded deformable multi-layer silicone VF replicas (labelled M5, MRI and EPI) are considered. The replicas are depicted in Fig. 1(a) 12. They differ with respect to their shape and layer composition so that they mimic the multi-layer structure and geometry of the human VF's with different degrees of complexity. Each VF replica consists of a left and right VF placed face-to-face in the transverse plane. The molded VF replicas are briefly detailed in Section 2, In previous studies [12, 13, these three replicas were used to study the effect of vertical tilting of a single VF on their autooscillation. Concretely, the right VF was kept in place whereas the posterior edge of the left VF was tilted in the medio-sagittal plane towards the superior direction as shown in Fig. 1(b) and in Fig. 1(c) The resulting vertical tilting is parameterised by angular asymmetry angle $\alpha$. Imposing angular asymmetry in the range from $0^{\circ}$ up to $25^{\circ}$ results in a vertical level difference up to a few $\mathrm{mm}$ [12, which is of the same order of magnitude as observed in patients suffering from UVFP [2, 3. Furthermore, vertical tilting of a VF causes left-right VF positioning asymmetry whereas left-right VF tension and shape symmetry is maintained.

In [12, the upstream pressure driving VF auto-oscillation was analysed for 


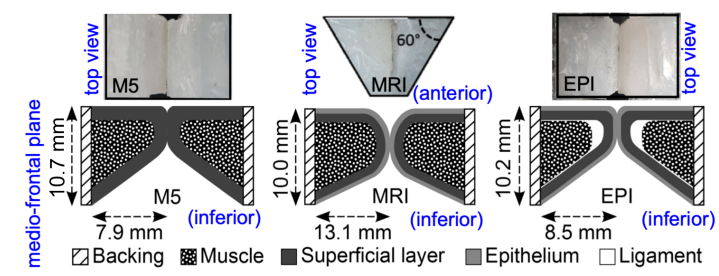

(a) deformable portion: shape and composition

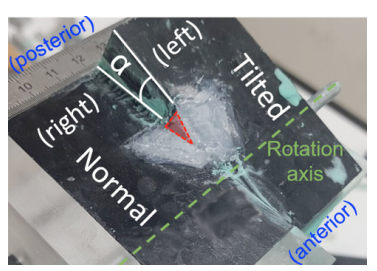

(b) tilting of rigid support

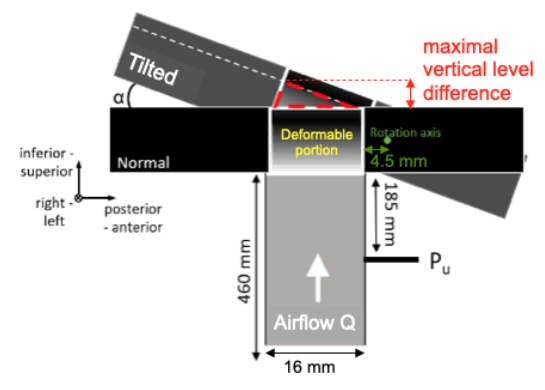

(c) overview fluid-structure interaction setup

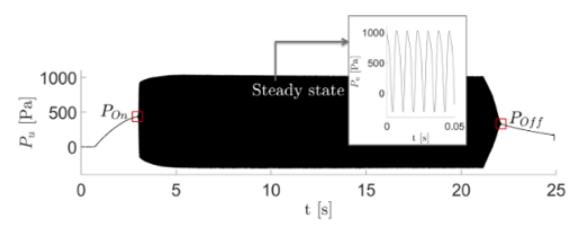

(d) measured $P_{u}(t)$

Figure 1: Illustration of deformable VF replicas and fluid-structure interaction setup: a) multi-layered deformable silicone VF replicas with increasing complexity for $\alpha=0^{\circ}$ [12]: twolayer M5, three-layer MRI and four-layer EPI. b) general view of imposed asymmetry angle $\alpha$, glottal gap associated with air leakage (red triangle) and fixed rotation axis for tilting of the left VF for the MRI replica with $\alpha=20^{\circ}$. c) overview of the fluid-structure interaction setup [12] indicating the pressure tap to measure upstream pressure $P_{u}$ and maximal vertical difference associated with each imposed $\alpha$. d) example (EPI replica, $\alpha=0^{\circ}$ ) of measured $P_{u}(t)$ illustrating the pressure at oscillation onset $P_{O n}$, steady state oscillation and oscillation offset $P_{O f f}$.

different imposed angular asymmetry angles $\alpha$ using the setup schematically so illustrated in Fig. 1(c). It was found that both the oscillation onset characteristics as well as common voice quality measures are affected. For instance, a decrease of the oscillation frequency and an increase of the oscillation onset threshold pressure $\left(P_{O n}\right)$ with increasing $\alpha$ was observed. The gradual loss of VF's contact with $\alpha$, inducing increased glottal air leakage, was pointed out to catalyse these tendencies.

In [13, high-speed (HS) imaging of the VF's for different $\alpha$ was performed 
in order to directly observe the vibrating VF's. Local image features, exploiting HS videokymographic (VK) line-scans [14, were quantified during steady state oscillation (Fig. 1(d)). Left-right vibration asymmetry parameters showed that the normal VF (right VF in Fig. 1(b)] entrains the movement of the tilted VF (left VF in Fig. 1(b)p. This left-right vibration asymmetry caused the mucosal wave velocity in the tilted VF to be lower than the one in the normal VF.

Local VK line-scan analysis [13] showed the influence of angular asymmetry on VF vibration. In this work, it is sought to further investigate sustained steady state $\mathrm{VF}$ vibration without $\left(\alpha=0^{\circ}\right)$ and with $\left(\alpha>0^{\circ}\right)$ angular asymmetry by analysing high-speed (HS) image sequences of the vibrating VF's. Instead of focusing on local spatial features or line scans [13, 15, 17, 18] it is sought to assess simultaneously temporal as well as spatial information of the global VF's auto-oscillation. A dynamic vibration mode decomposition (DMD) of the observed vibration snapshots is applied as other VF vibration mode decomposition methods such as the empirical eigenfunction analysis 19 do not inform on the temporal mode dynamics. It is aimed to evaluate how the effect of angular asymmetry on the VF's auto-oscillation affects the spatial and temporal vibration mode features.

\section{Deformable silicone VF replicas}

The M5, MRI and EPI replicas illustrated in Fig. 1(a) are molded so that their elasticity is constant as detailed in [12. They consist of respectively two (M5), three (MRI) or four (EPI) silicone-based molding layers and a backing layer attaching it to a rigid support, i.e. region outside of the top view frames shown in Fig. 1(a), The M5 replica is a two-layer (muscle and superficial layer) reference model following the so called M5 geometrical VF model [20. The MRI replica has a more realistic geometry derived from magnetic resonance imaging data of a human VF [21, 22]. It has a three-layer structure by adding a third 
thin and stiff surface layer representing the epithelium to the two-layer structure of the M5 replica. The EPI replica is obtained by inserting an extremely soft layer between the muscle and the superficial layer of the three-layer structure used for the MRI replica [11. The EPI replica cast is inspired on the geometrical M5 model [11] so that the geometry of the EPI replica approximates the geometry of the M5 replica as illustrated in Fig. 1. Young moduli for each layer of these VF replicas are given in 12] (Table I). The preset abduction along the left-right direction for angular asymmetry angle $\alpha=0^{\circ}$ is shown in Fig. 1(a). 75 The left and right VF are in full contact along the posterior-anterior direction. As $\alpha$ is increased, the degree of contact decreases as illustrated in Fig. 1(b) and Fig. 1(c) The preset (no airflow) degree of vocal fold contact $\mathcal{G}$ (in percentage) associated with each replica as a function of angular asymmetry angle $\alpha$ is indicated in Table 1 [12].

\section{High-speed imaging of VF auto-oscillation}

The experimental setup and flow supply used to generate the FS interaction underlying the VF auto-oscillation is schematically depicted in Fig. 1(c). The setup is similar to the one described in [12,13. Airflow, characterised by volume flow rate $Q$ (sampling frequency $0.5 \mathrm{kHz}$ ), is applied along the inferior-superior direction as depicted in Fig. 1(c) and in Fig. 2 The pressure difference driving the VF's auto-oscillation corresponds to the measured upstream pressure $P_{u}$ (sampling frequency $10 \mathrm{kHz}$ ). All experiments are performed with a mean upstream pressure $\bar{P}_{u}$ set just above $(<50 \mathrm{~Pa})$ the oscillation onset threshold $P_{O n}$ so that $\bar{P}_{u} \gtrsim P_{O n}$. An example of the measured upstream pressure as a function of time is plotted in Fig. 1(d) indicating the pressure at oscillation onset $P_{O n}$, oscillation offset $P_{O f f}$ and during sustained steady state oscillation. In this work, steady state oscillation is analysed. Assessed flow conditions and associated fundamental frequency $f_{o}$ obtained from a Fourier analysis of the upstream pressure $P_{u}$ during steady oscillation as outlined in [12] are given in 
Table 1: Assessed experimental conditions: mean upstream pressure $\bar{P}_{u}(\mathrm{~Pa})$, mean volume flow rate $\bar{Q}(\mathrm{~L} / \mathrm{min})$, fundamental frequency $f_{o}(\mathrm{~Hz})$ and degree of left-right $\mathrm{VF}$ contact $\mathcal{G}$ $(\%)$.

\begin{tabular}{cc||c|c|c|c|c}
\hline & & $\alpha=0^{\circ}$ & $\alpha=4^{\circ}$ & $\alpha=10^{\circ}$ & $\alpha=16^{\circ}$ & $\alpha=20^{\circ}$ \\
\hline \hline \multirow{2}{*}{ M5 } & $\bar{P}_{u}$ & 1106 & 1174 & 1015 & 1076 & 1488 \\
& $\bar{Q}$ & 13.8 & 14.1 & 19.4 & 48.6 & 63.4 \\
& $f_{o}$ & 138 & 138 & 138 & 134 & 126 \\
& $\mathcal{G}$ & 100 & 100 & 73 & 38 & 23 \\
\hline \multirow{2}{*}{ MRI } & $\bar{P}_{u}$ & 1251 & 1279 & 1180 & & 1373 \\
& $\bar{Q}$ & 25.5 & 37.7 & 39.1 & & 66.7 \\
& $f_{o}$ & 144 & 141 & 128 & - & 118 \\
& $\mathcal{G}$ & 100 & 100 & 69 & & 21 \\
\hline \multirow{2}{*}{ EPI } & $\bar{P}_{u}$ & 438 & 407 & 435 & & 556 \\
& $\bar{Q}$ & 22.4 & 23.9 & 26.0 & & 51.5 \\
& $f_{o}$ & 129 & 127 & 114 & & 93 \\
& $\mathcal{G}$ & 100 & 65 & 10 & & 0 \\
\hline
\end{tabular}

Table 1 .

This implies that the imposed $\bar{P}_{u}$ differs between assessed conditions as $P_{O n}$ depends on the used VF replica (M5, MRI or EPI) as well as on the imposed angular asymmetry angle $\alpha$ (Fig. 7 in [12]). Assessed $\alpha$ (from $0^{\circ}$ up to $20^{\circ}$ ) and corresponding vertical level differences $\bar{h}$ (from $0 \mathrm{~mm}$ up to $3 \mathrm{~mm}$ as reported for UVFP patients 2, 3]) are shown in the frame of Fig. 2 for each VF replica. The overall vertical level difference $\bar{h}$ in absence of airflow is obtained from the experimentally imposed $\alpha$ following a geometrical reasoning as outlined in [13] (section 2.3 therein) and yields half of the maximum vertical level difference along the triangular air leakage area indicated in Fig. 1(c)

A single high speed (HS) camera (Keyence VW-9000, shutter time $4 \mu$ s and resolution $240 \mathrm{px} \times 320 \mathrm{px}$ ) is placed in the medio-frontal plane in order to acquire instantaneous images of the VF's steady state auto-oscillation as outlined in [13. The imaging frame rate $f_{s}$ is set to $4 \mathrm{kHz}$ so that images are gathered ev- 


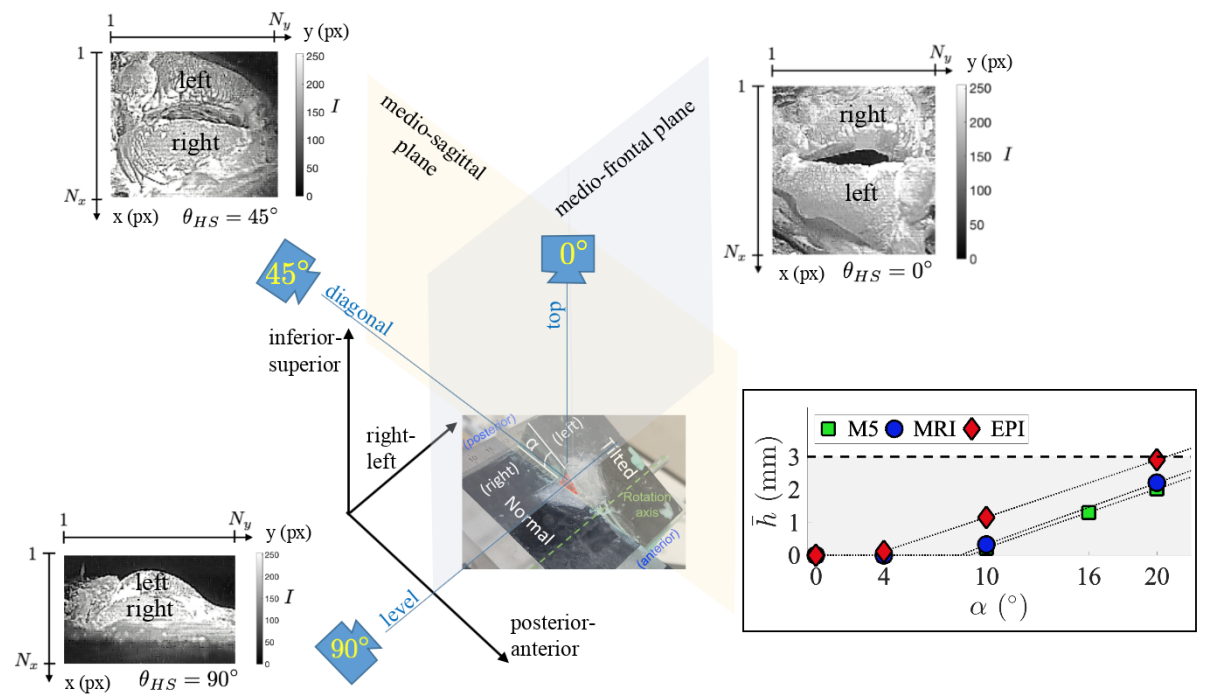

Figure 2: Overview of experimental setup with HS camera view angles 13: $\theta_{H S}=0^{\circ}$ (top view), $\theta_{H S}=45^{\circ}$ (diagonal view) and $\theta_{H S}=90^{\circ}$ (level view). Initial (no airflow) vertical level difference $\bar{h}$ resulting from varying angular asymmetry $\alpha$ between $0^{\circ}$ and $20^{\circ}$ for each VF replica (M5, MRI and EPI) is framed. The shaded area $0 \leq \bar{h} \leq 3 \mathrm{~mm}$ covers values reported for patients [2, 3. Snapshots during VF vibration to illustrate captured images $\mathbf{I m}_{k}=I\left(x, y ; t_{k}\right)$ at instant $t_{k}$.

ery $\Delta t$ seconds at time instants $t_{k}=k \cdot \Delta t$ with $\Delta t=1 / f_{s}$ yielding $0.25 \mathrm{~ms}$ and $k$ denoting the frame number in the image sequence. This temporal accuracy corresponds to $\leq 3.8 \%$ of the period for oscillation frequencies $\leq 150 \mathrm{~Hz}$, which is pertinent for the primary oscillation frequency of each assessed experimental condition regardless of the used VF replica and imposed asymmetry angle $\alpha$ as shown in [12, 13] and in Table 1. For oscillation frequencies $\leq 750 \mathrm{~Hz}$, the temporal accuracy decreases to $\leq 15 \%$ of the oscillation period.

The VF vibration during auto-oscillation is observed non-simultaneously from three distinct single view angles $\theta_{H S}$, defined as the angle between the observation direction and the medio-sagittal plane: top view $\theta_{H S}=0^{\circ}$, diagonal view $\theta_{H S}=45^{\circ}$ and level view $\theta_{H S}=90^{\circ}$. Camera positions and corresponding view angles $\theta_{H S}$ are depicted in Fig. 2. 
Acquired images at each instant $t_{k}$ are two dimensional grayscale intensity matrices $\mathbf{I m}_{k}=I\left(x, y ; t_{k}\right)$ with $x$ and $y$ indicating the pixel position in the image and where subscript $k$ denotes time instant $t_{k}$. It follows that $1<x \leq N_{x}$ and $1<y \leq N_{y}$, with $N_{x}=240$ and $N_{y}=320$ determined by the camera resolution, so that each image contains $n=76800$ pixel positions.

130

For each assessed condition, defined by both the VF replica (M5, MRI or EPI) and imposed angular asymmetry angle $\alpha, 1 \mathrm{~s}$ of steady-state autooscillation is analysed. For each replica and view angle, the system dynamics f of the fluid-structure interaction during auto-oscillation is represented as a 135 function of asymmetry angle $\alpha$ as

$$
\frac{\mathrm{d} \mathbf{s}}{\mathrm{d} t}=\mathbf{f}(\mathbf{s}, t ; \alpha),
$$

where $\mathbf{s} \in \mathbb{R}^{n}$ represents the state of the system with dimension $n \gg 1$ at time t. In discrete-time representation Eq. (1) becomes

$$
\mathbf{s}_{k+1}=\mathbf{F}\left(\mathbf{s}_{k} ; \alpha\right)
$$

as system states are denoted $\mathbf{s}_{k}=\mathbf{s}\left(t_{k}\right)$, where subscript $k$ denotes again time instant $t_{k}$.

140

Gathered image vectors $\mathbf{I}_{k}=\operatorname{vec}\left(\mathbf{I m}_{k}\right)$, where $\operatorname{vec}(\cdot)$ is the vectorised operator, provide snapshots of the system at times $t_{k}$, so that

$$
\mathbf{I}_{k}=\mathbf{g}\left(\mathbf{s}_{k}\right)
$$

with $k=1, \ldots, m$ where $m$ denotes the total number of snapshots in each image sequence. Analysing $1 \mathrm{~s}$ of steady-state auto-oscillation corresponds to an image sequence of $m=4000$ subsequent snapshots. It is further assumed that at each time instant $t_{k}$ the state corresponds to the measured image so that

$$
\mathbf{s}_{k}=\mathbf{I}_{k}
$$


holds. The system dimension $n$ corresponds then to the number of pixel positions. Initial conditions are prescribed as $\mathbf{I}_{1}=\mathbf{I}\left(t_{1}\right)$ at time $t_{1}=0 \mathrm{~s}$.

\section{Dynamic mode decomposition}

The temporal and spatial decomposition of the global VF auto-oscillation is assessed by a dynamic mode decomposition (DMD) of the gathered image sequences [23, 24].

The DMD framework is model free so that the system dynamics is estimated from each measured image sequence only. Assuming a locally linear system dynamics

$$
\frac{\mathrm{d} \mathbf{s}}{\mathrm{d} t}=\mathbf{X} \mathbf{s}
$$

the solution is given as

$$
\mathbf{s}(t)=\sum_{j=1}^{n} \phi_{j} \exp \left(\omega_{j} t\right) b_{j},
$$

where $\phi_{j}$ and $\omega_{j}$ denote the eigenvectors and eigenvalues of the system matrix $\mathbf{X}$ and where the coefficients $b_{j}$ are the coordinates of $\mathbf{I}\left(t_{1}\right)$ in the eigenvector basis [25]. In matrix notation this becomes

$$
\mathbf{s}(t)=\boldsymbol{\Phi} \exp (\boldsymbol{\Omega} t) \mathbf{b}
$$

where $\boldsymbol{\Omega}=\operatorname{diag}(\omega)$ is a diagonal matrix whose entries are the eigenvalues $\omega_{j}$, $\mathbf{\Phi}$ is a matrix whose columns are the eigenvectors $\phi_{j}$ and $\mathbf{b}$ is a column vector of the coefficients $b_{j}$.

Using the assumption $\mathbf{s}_{k}=\mathbf{I}_{k}$ expressed in Eq. (4), the discrete-time analogous of Eq. (5) is

$$
\mathbf{I}_{k+1}=\mathbf{A} \mathbf{I}_{k}
$$


with

$$
\mathbf{A}=\exp (\mathbf{X} \Delta t)
$$

The solution is then given as

$$
\mathbf{I}_{k}=\sum_{j=1}^{r} \phi_{j} \lambda_{j}^{k-1} b_{j}
$$

with $\phi_{j}$ and $\lambda_{j}$ the eigenvectors and eigenvalues of the discrete time map $\mathbf{A}$ and with $b_{j}$ the coefficients of $\mathbf{I}_{1}$ in the reduced eigenvector basis of rank $r \leq n$. In matrix notation this becomes

$$
\mathbf{I}_{k}=\mathbf{\Phi} \boldsymbol{\Lambda}^{k-1} \mathbf{b}
$$

with $\boldsymbol{\Phi}$ and $\mathbf{b}$ defined in Eq. 7 and $\boldsymbol{\Lambda}^{k-1}=\operatorname{diag}\left(\left\{\lambda_{j}^{k-1}\right\}_{j}\right)$ is a diagonal matrix whose entries are the eigenvalues $\lambda_{j}$ raised to the power $k-1$. The exponent $k$ indicates the time instant $t_{k}$ of the image snapshot. The initial condition is given as $\mathbf{I}_{1}=\mathbf{\Phi} \mathbf{b}$.

The sought spatial analysis of the global auto-oscillation is thus provided by the DMD modes $\phi_{j}$ given by the eigenvectors of $\mathbf{A}$. The sought temporal analysis of each DMD mode is provided by the corresponding eigenvalue $\lambda_{j}$ of A so that from Eq. 6 and Eq. 10

$$
\omega_{j}=\frac{\ln \left(\lambda_{j}\right)}{\Delta t}
$$

holds and the mode frequency is given by $f_{j}=\operatorname{Im}\left(\omega_{j}\right) / 2 \pi$ and its growth rate by $\operatorname{Re}\left(\omega_{j}\right)$ so that $\operatorname{Re}\left(\omega_{j}\right) \leq 0$ holds for stable modes. The DMD eigenvalue spectrum is then obtained by plotting for each mode $j$ its frequency $\operatorname{Im}(\omega) / 2 \pi$ as a function of its growth rate $\operatorname{Re}(\omega)$ where $\omega=\omega_{j}$. The amplitude of each mode corresponds to the associated coefficient $b_{j}$ in $\mathbf{b}$.

It follows that the superposition of the DMD modes, weighted by their amplitude and temporal behavior results in a spatio-temporal representation of 

are presented in Section 6 .

\section{Temporal vibration analysis: DMD eigenvalue spectra}

The temporal behavior of the vocal folds vibration for different VF replicas

the full VF auto-oscillation dynamics. The dimension of the representation depends on the eigenvector basis of rank $r$ taken into account. In the current work, DMD analysis is aimed and not reconstruction or data reduction so that mode selection (or rank $r$ ) is not crucial. Instead, DMD eigenvalue spectra for modes with oscillation frequencies up to $1600 \mathrm{~Hz}$ are presented in Section 5 and spatial DMD mode patterns for modes with oscillation frequencies up to $750 \mathrm{~Hz}$ and imposed angular asymmetry angles $\alpha$ is analysed considering the DMD eigenvalue spectra. Spectra of stable vibration modes for all three replicas and top view angle $\theta_{H S}=0^{\circ}$ are plotted in Fig. 3 .

All spectral branches originate with a non-oscillation mode $(0 \mathrm{~Hz}, j=1)$ associated with the zero eigenvalue at the origin $(\operatorname{Re}(\omega)=0$ and $\operatorname{Im}(\omega) / 2 \pi=0)$. This non-oscillation mode expresses the influence of the mean flow along the inferior-superior direction on the VF's vibration pattern. Stable branches with least decay are identified as main spectral branches. Dominating oscillation modes $(j=2 \ldots 6)$ on the main spectral branches with frequencies up to $\approx 750 \mathrm{~Hz}(\operatorname{Im}(\omega) / 2 \pi \leq 750)$ are indicated with filled symbols in Fig. 3. Both the non-oscillation mode and most dominating oscillation modes decay slowly as their real parts approximate zero $(\operatorname{Re}(\omega) \leqq 0)$. The real part of the eigenvalue $\operatorname{Re}(\omega)$ decreases as the mode frequency increases, typically above $\approx 750 \mathrm{~Hz}$ or $\operatorname{Im}(\omega) / 2 \pi>750$ for the main spectral branches, so that these modes decay mode rapidly and hence contribute less to observed temporal vibration patterns. It is noted that side branches might occur such as e.g. observed for $\alpha=0^{\circ}$ and $\alpha=10^{\circ}$ in Fig. 3(a) It is noted that primary oscillation (PO mode, mode $j=2$ ) frequencies obtained from the DMD eigenvalue spectra matches with $f_{o}$ 

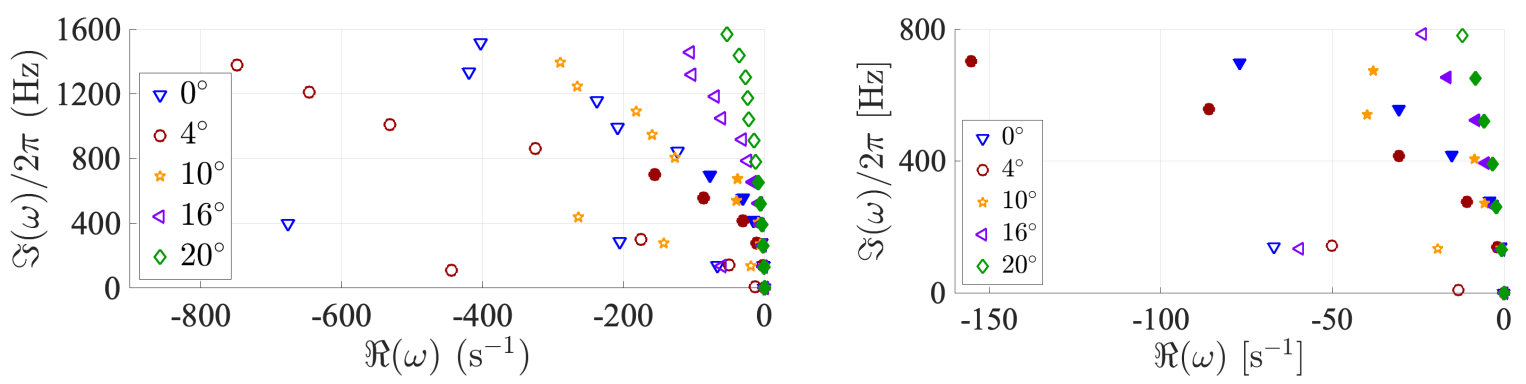

(a) M5: spectrum and zoom
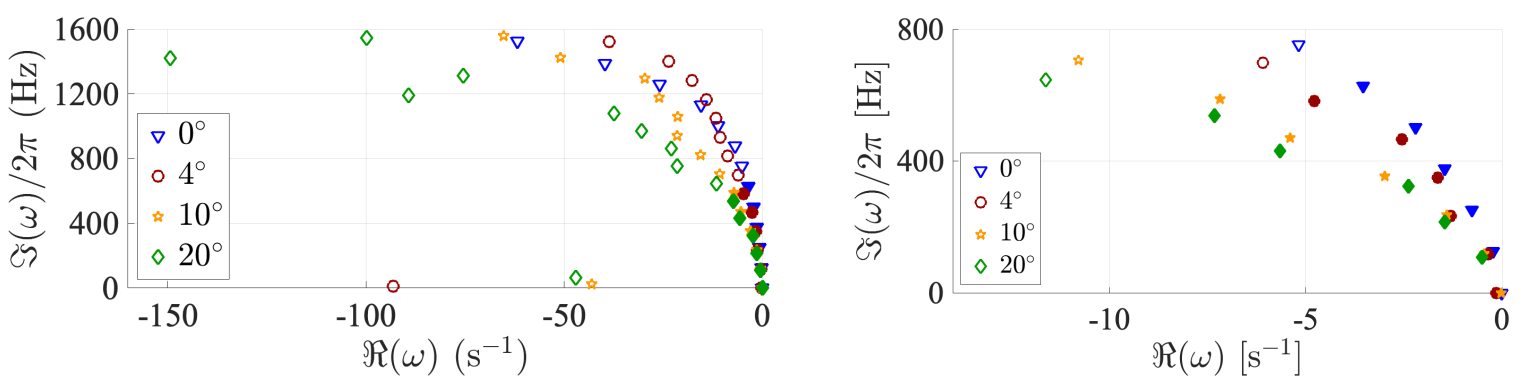

(b) MRI: spectrum and zoom
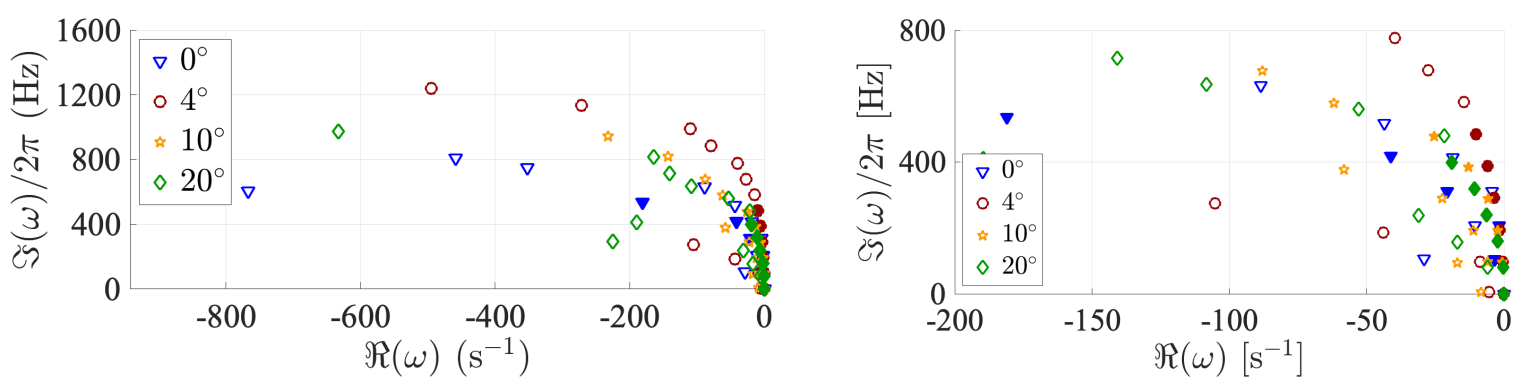

(c) EPI: spectrum and zoom

Figure 3: DMD eigenvalue spectra (left) and zoom (right) for top view $\theta_{H S}=0^{\circ}$ and different asymmetry angles $\alpha$ (symbols) for all three VF replicas: a) M5, b) MRI and c) EPI. Filled symbols indicate dominating modes with frequency $\operatorname{Im}(\omega) / 2 \pi \leq 750 \mathrm{~Hz}$.

values reported in Table 1 and with previously reported values [12, 13.

Despite these similar tendencies for all VF replicas, Fig. 3 shows that the type/structure of VF replica affects the influence of angular asymmetry angle 
$\alpha$ on the eigenvalue spectra. This is most obvious when comparing eigenvalue spectra obtained for the M5 replica with spectra obtained for the MRI or EPI replica.

Increasing the angular asymmetry from $\alpha=0^{\circ}$ to $\alpha=4^{\circ}$ either decreases (MRI/EPI) or increases (M5) the decay of high frequency modes. Indeed, tilting a VF from $\alpha=0^{\circ}$ to $\alpha=4^{\circ}$ results in either steeper $(\operatorname{Re}(\omega)$ increases for MRI/EPI) or more sloped $(\operatorname{Re}(\omega)$ decreases for M5) spectral branches. Therefore, in terms of the number of non-decaying stable oscillation modes, introducing a slight angular asymmetry ( $\bar{h} \ll 1 \mathrm{~mm}$ Fig. 2 ) either enriches (MRI/EPI) or strips (M5) the vibration pattern.

Further increasing the angular asymmetry angle from $\alpha=4^{\circ}$ reveals the opposite tendency as mode decay either decreases $(\operatorname{Re}(\omega)$ increases, M5) or increases ${ }_{220}(\operatorname{Re}(\omega)$ decreases, MRI/EPI). So that in terms of the number of non-decaying stable oscillation modes, the vibration pattern either enriches (M5) or recedes (MRI/EPI) with $\alpha \geq 4^{\circ}$.

Given the geometry and multi-layer composition of the used VF replicas (Fig. 1(a) and Section 2p, observed eigenvalue spectral differences are attributed to the presence (MRI/EPI) or absence (M5) of a thin and stiff surface layer representing the epithelium. As perfect angular symmetry $\left(\alpha=0^{\circ}\right)$ is unlikely to occur in real life, the found robustness of the MRI/EPI VF replica to small angular asymmetries ( $\alpha=4^{\circ}$ in Fig. 2 ) supports the hypothesis that adding an epithelium-like layer alters silicone VF replicas from a vibratory point of view. In addition, it provides evidence of the importance of this layer for normal VF vibration. Larger angular asymmetries $\left(\alpha>4^{\circ}\right.$ in Fig. 3) mimic pathological VF vibration as all replicas become prone to glottal air leakage due to vertical level difference $(\bar{h}>0 \mathrm{~mm}$ in Fig. 22 [12. Increased mode decay, only observed for the MRI/EPI replica and not for the M5 replica, is consistent with impaired oscillation and reduced vibration quality associated with glottal insufficiency reported for human speakers. Thus for the assessed silicone VF replicas, it is hypothesized that from a vibration point of view the presence of an epitheliumlike layer results in more reasonable vibration patterns. 
From Fig. 3 is seen that the effect of $\alpha$ on mode decay is most obvious for higher frequency modes (typically $>750 \mathrm{~Hz}$ ). Therefore, it might be of interest to investigate higher frequency vibration modes as potential clinical markers for voice pathology studies such as UVFP.

The described changes in decay rate with $\alpha$ can be partly explained by changes in structural damping. Structural damping is likely to increase (or decrease) as the duration of vocal fold contact along the inferior-superior direction inside each glottal cycle increases (or decreases). This duration was quantified for the assessed VF replicas in [13. It follows that structural damping either increases (MRI/EPI) or decreases (M5) with $\alpha$ for $\alpha \geq 4^{\circ}$ as vocal fold contact 250 was found to increase (MRI/EPI) or decrease (M5) with $\alpha$.

So far, spectral similarities, with respect to the influence of angular asymmetry angle $\alpha$, between the MRI and the EPI replica were pointed out. Nevertheless, comparing Fig. 3(b) and Fig. 3(c) shows for all $\alpha$ that the onset of vibration mode decay occurs at lower mode frequencies for the EPI than for the MRI replica. This observation is again partly explained considering structural damping. As the oscillation frequency of the dominant oscillation mode for the EPI replica $(\approx 120 \mathrm{~Hz}[13])$ is lower than the one observed for the MRI replica $(\approx 140 \mathrm{~Hz}[13$ ] $)$ the contribution of structural damping to mode decay during 260 a similar relative portion of the glottal cycle will be greater for the EPI than for the MRI replica. Hence, mode decay for the EPI replica is enhanced compared to the MRI replica. The different relative closure duration during each glottal cycle between both VF replicas is due to differences in their shape and their composition underneath the epithelium layer as outlined in Fig. 1(a) and Section 2

Fig. 4 illustrates, for the EPI replica, that varying the camera view angle $\theta_{H S}$ from top view $\theta_{H S}=0^{\circ}$ to either diagonal view $\theta_{H S}=45^{\circ}$ or level view $\theta_{H S}=90^{\circ}$, shown in Fig. 2, preserves the general eigenvalue spectral 


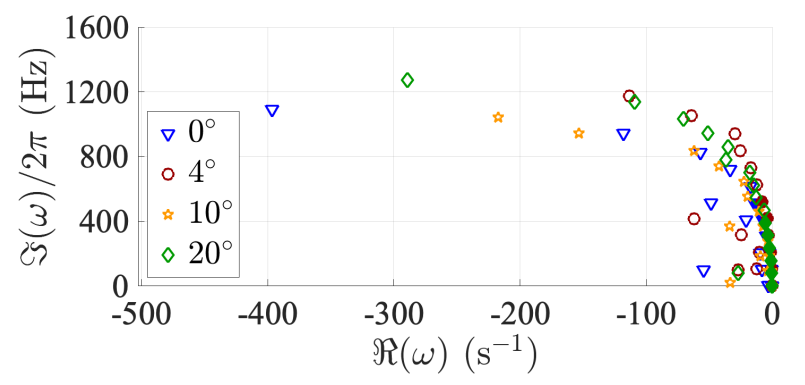

(a) EPI, $\theta_{H S}=45^{\circ}$

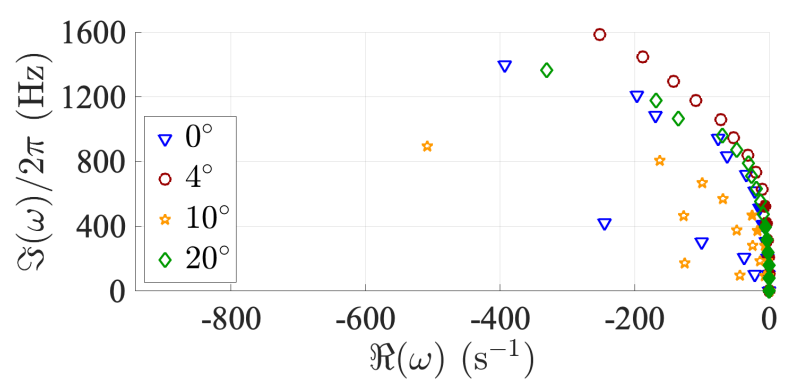

(b) EPI, $\theta_{H S}=90^{\circ}$

Figure 4: DMD eigenvalue spectra for the EPI replica and different asymmetry angles $\alpha$ (symbols) for: a) diagonal view $\theta_{H S}=45^{\circ}$ and b) level view $\theta_{H S}=90^{\circ}$. Filled symbols indicate dominating modes with oscillation frequency $\operatorname{Im}(\omega) / 2 \pi \leq 750 \mathrm{~Hz}$.

tendencies observed for top view $\theta_{H S}=0^{\circ}$ (Fig. 3(c) $)$. Nevertheless, whereas no significant changes to the decay of dominant oscillation modes (filled symbols) are observed, the decay of higher frequency oscillation modes (non-filled symbols) slows down as the camera view angle is tilted towards higher $\theta_{H S}$. As higher frequency modes have more complex vibration patterns, tilted views $275\left(\theta_{H S}=45^{\circ}, \theta_{H S}=90^{\circ}\right)$ allow a better observation of these modes than the top view $\theta_{H S}=0^{\circ}$ as, in addition to the transverse plane, they allow observation of the modes along the medio-sagittal plane. 


\section{Spatial vibration analysis: DMD modes}

280 indicated by filled symbols in Fig. 3 and Fig. 4 , are arranged $(j=1 \ldots 6)$ according to increasing frequency.

\subsection{Mean flow modes}

Examples of non-oscillation spatial modes, corresponding to the zero eigenvalue at the origin of the eigenvalue spectra (mode $j=1$ ), are illustrated in Fig. 5. These modes are due to the mean flow during the fluid-structure interaction along the inferior-superior direction and depend on the imposed asymmetry angle $\alpha$ as the resulting glottal air leakage affects the mean glottal flow.
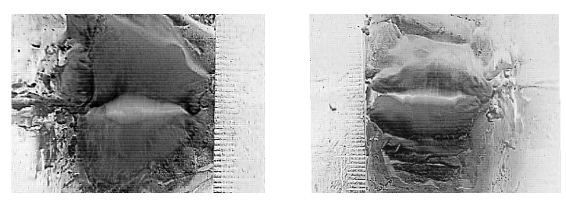

(a) MRI, $\alpha=4^{\circ}$

(b) EPI, $\alpha=0^{\circ}$

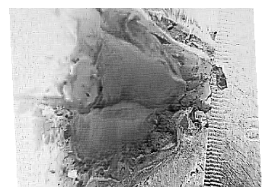

(c) MRI, $\alpha=20^{\circ}$

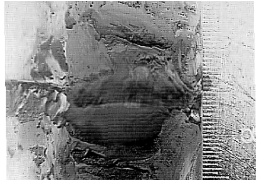

(d) EPI, $\alpha=10^{\circ}$

Figure 5: Illustration of spatial mean flow or non-oscillation mode patterns $\phi_{j}$ for $j=1$ scaled between 0 (white, invariant mode region) and 1 (black, most variant mode region) for top view $\theta_{H S}=0^{\circ}$.

Non-oscillation mode amplitudes $b_{1}$ are plotted in Fig. 6. All amplitudes are normalised by the amplitude of the PO mode $(j=2)$ associated with the lowest stable oscillation frequency for $\alpha=0^{\circ}$ denoted as $b_{2,0^{\circ}}$. As normalised values exceed unity for each assessed configuration, regardless of the used VF replica 

the eigenvalue spectra.

For all view angles $\theta_{H S}$ and angular asymmetry angles $\alpha$, the imprint of the mean flow is least pronounced for the EPI replica as normalised ampli-

300

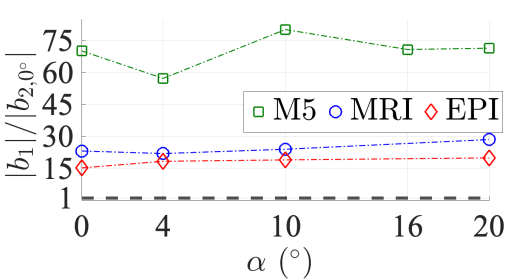
(b) $\theta_{H S}=45^{\circ}$
(a) $\theta_{H S}=0^{\circ}$

Figure 6: Normalised non-oscillation mode amplitude magnitude as a function of asymmetry angle $\alpha$ for all VF replicas (M5, MRI and EPI): a) top view $\theta_{H S}=0^{\circ}$, b) diagonal view $\theta_{H S}=45^{\circ}$. The unity amplitude of the primary oscillation mode for $\alpha=0^{\circ}$ is indicated (horizontal dashed line). 


\subsection{Oscillation modes}

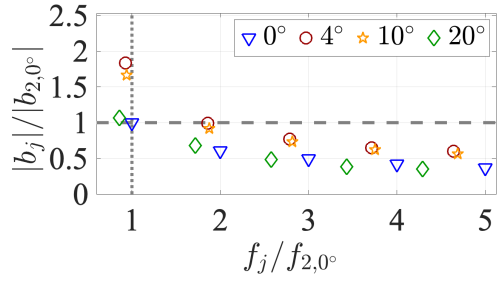

(a) MRI, $\theta_{H S}=0^{\circ}$

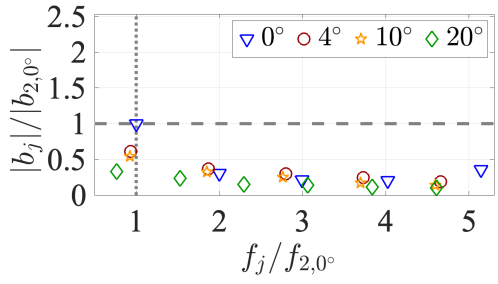

(c) EPI, $\theta_{H S}=0^{\circ}$

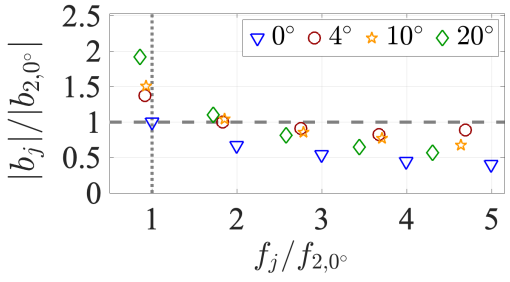

(b) MRI, $\theta_{H S}=45^{\circ}$

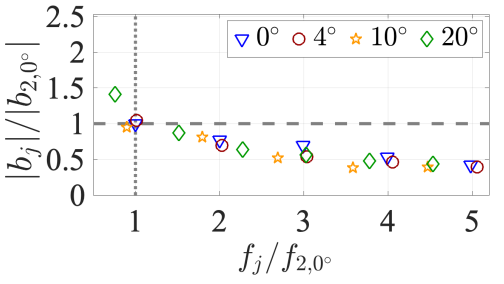

(d) EPI, $\theta_{H S}=45^{\circ}$

Figure 7: Oscillation mode $(j=2 \ldots 6)$ amplitudes as a function of their oscillation frequency and different asymmetry angles $\alpha$ (symbols) with top view $\theta_{H S}=0^{\circ}$ and diagonal view $\theta_{H S}=45^{\circ}$ for the MRI (a,b) and for the EPI replica (c,d). Amplitudes and frequencies are normalized with respect to values of the primary oscillation (PO) mode $(j=2)$ with $\alpha=0^{\circ}$ indicated with a dashed horizontal and dotted vertical line respectively.

The normalised amplitudes of five oscillation modes $(j=2 \ldots 6)$ for the MRI and EPI replicas, indicated by the filled symbols away from the origin in the eigenvalue spectra of Fig. 3 and Fig. 4, are plotted in Fig. 7 as a function of their normalised mode frequency. Plotted quantities are normalised with respect to the amplitude $b_{2,0^{\circ}}$ and the frequency $f_{2,0^{\circ}}$ of the primary oscillation mode (PO mode, $j=2$ ) at $\alpha=0^{\circ}$. Amplitudes obtained with top view $\theta_{H S}=0^{\circ}$ (Fig. 7(a) and Fig. 7(c) and with diagonal view $\theta_{H S}=45^{\circ}$ (Fig. 7(b) and Fig. 7(d) are plotted.

The PO mode, whose frequency corresponds to the fundamental frequency 325 characterising voiced speech utterances, has the largest amplitude for each angular asymmetry angle $\alpha$. It is noted that the PO mode frequencies match with 
auto-oscillation frequencies previously reported either from VK image analysis [13] or from upstream pressure flow analysis [12]. Therefore, current results confirm the reported decrease of the PO mode frequency with increasing angular asymmetry $\alpha$ so that $f_{2} / f_{2,0^{\circ}} \leq 1$ holds as seen in Fig. 7 .

Higher order post-PO modes obtained for $j=3 \ldots 6$, exhibit oscillation frequencies near the harmonics of the PO mode frequency $f_{2}$ for all angular asymmetry angles $\alpha$ as $f_{j} / f_{2} \approx j-1$ holds. Discrepancies between post-PO mode frequencies and PO mode harmonics are insignificant considering the temporal accuracy associated with the used image frame rate (section 3).

As observed for the non-oscillatory mean flow mode $(j=1)$, normalised oscillation mode amplitudes obtained with $\theta_{H S}>0^{\circ}$ are mostly larger than those obtained with top view angle $\theta_{H S}=0^{\circ}$ as mode patterns develop along both the horizontal transverse as well as along the medio-sagittal plane. From Fig. 7 is seen that, in general, normalised mode amplitudes reduce with $j$ for each view angle $\theta_{H S}$ and angular asymmetry angle $\alpha$.

Spatial oscillation modes $\phi_{j}$ for $j=2 \ldots 6$ (columns) of the MRI replica are illustrated for top view $\theta_{H S}=0^{\circ}$ in Fig. 8(a) and for diagonal view $\theta_{H S}=45^{\circ}$ in Fig. 8(b) Spatial mode activity is colour-coded between white (invariant mode regions) and black (most variant mode regions). The imposed asymmetry angle $\left(\alpha=4^{\circ}\right.$ or $\left.\alpha=20^{\circ}\right)$ is indicated in front of each row and the oscillation mode order $(j=2 \ldots 6)$ is indicated on top of each column.

350

Increasing mode order $j$ changes the spatial mode with respect to its extent and with respect to the node pattern. In the vicinity of the glottal aperture (within the frames in Fig. 8) typical higher order mode patterns are observed as the number of nodes increases with $j$. For small angular asymmetry angles, e.g. ${ }_{355} \alpha=4^{\circ}$ in Fig. 8, mode patterns develop similarly along the posterior-anterior direction and occupy both VF surfaces as tilting does not alters the elasticity of each VF. For large angular asymmetry angles, e.g. $\alpha=20^{\circ}$ in Fig. 8, spatial 

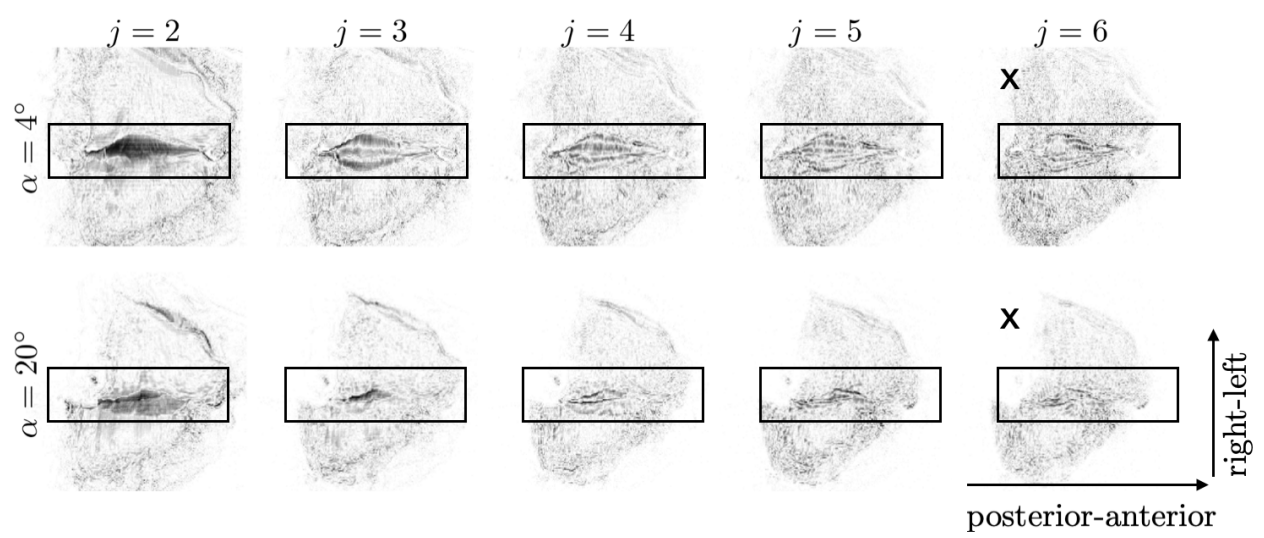

(a) $\theta_{H S}=0^{\circ}$
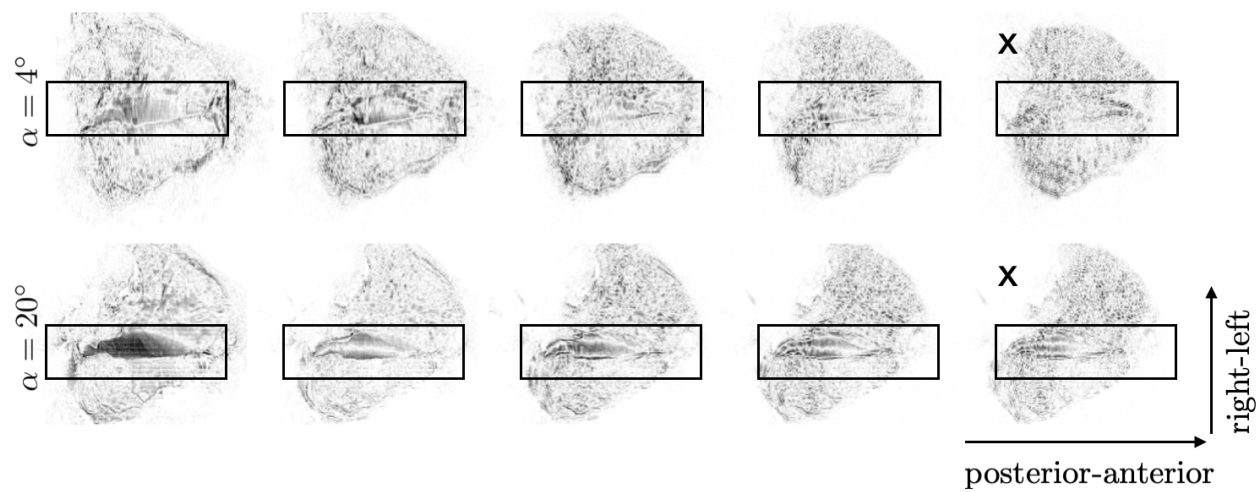

(b) $\theta_{H S}=45^{\circ}$

Figure 8: Spatial oscillating mode patterns $\phi_{j}$ for $j=2 \ldots 6$ (columns) scaled between 0 (white, invariant mode region) and 1 (black, most variant mode region) for the MRI replica at $\alpha=4^{\circ}$ and $\alpha=20^{\circ}$ (rows): a) top view $\theta_{H S}=0^{\circ}$, b) diagonal view $\theta_{H S}=45^{\circ}$. The glottal aperture region is framed. Posterior side of the left VF is tilted as illustrated for $j=6(\times)$.

modes reflects the loss of full VF contact along the posterior-anterior direction. It is observed that a surface region extending from the posterior edge of the tilted VF (illustrated for $j=6$ with a cross $(\times)$ in Fig. 8) is no longer part of the spatial mode pattern. Moreover, it is noted that the area of the excluded surface region increases with $j$. Near the glottal aperture the spatial mode pattern for large $\alpha$ depends on the used view angle $\theta_{H S}$. In the case of top view $\theta_{H S}=0^{\circ}$ 
in Fig. 8(a), reduced VF contact limits the complex node pattern. In the case of more clearly as the tilted VF (upper VF in Fig. 8(b) is directly observed. It is noted that the complex node pattern within the glottal aperture still extends along the full posterior-anterior direction and is still quasi-symmetrical between the normal and tilted VF given that it does not alters their elasticity.

370

\section{Conclusion}

Dynamic vibration mode decomposition in space and time of high-speed images of auto-oscillating silicone vocal folds replicas without and with vertical tilting is assessed. Temporal properties obtained from the eigenvalue spectra show that imposing vertical tilting affects mode decay in a way that for the assessed replicas is hypothesized to be associated with the presence or absence of a stiffer epithelium-like surface layer. The effect is observed most clearly for decaying higher order vibration modes with typical frequencies above $750 \mathrm{~Hz}$. The presence of a stiffer epithelium-like surface layer is also associated with a limited amplitude of the mean flow mode with respect to the amplitude of the primary oscillation mode. Vertical tilting reduces the spatial extent of the vibration mode near the tilted edge. On the other hand, the node pattern near the glottal aperture along the posterior-anterior direction is unaffected by vertical tilting as it does not affect the structural properties of the assessed VF replicas. mode properties as a function of vertical tilting needs further confirmation and investigation. Furthermore, it is of interest to vary besides the angular asymmetry angle also the elasticity of between the left and right vocal fold, as might occur in the case of UVFP, in order to consider the influence on the vibration modes. Moreover, current results encourage to investigate the potential interest of DMD analysis and resulting dynamic vibration mode properties, and in particular higher order vibration modes, as potential clinical markers derived 
from high-speed images of human vocal folds auto-oscillation, e.g. in the case of pathologies such as UVFP.

\section{Acknowledgements}

This work was partly supported by Full3DTalkingHead project (ANR-20CE23-0008-03) and JSPS International Research Fellowship (SP18205). Authors thank Dr. K. Ishimura (Ritsumeikan Univ., Japan) for experimental support.

\section{References}

[1] C. Rosen, C. Simpson, Operative techniques in laryngology, SpringerVerlag, 2008.

[2] K. Hong, K. Jung, Arytenoid appearance and vertical level difference between the paralyzed and innervated vocal cords, The Laryngoscope 111 (2001) 227-232.

[3] Y. Oyamada, E. Yumoto, K. Nakano, H. Goto, Asymmetry of the vocal folds in patients with vocal fold immobility, Arch Otolaryngol head neck surg 131 (2004) 399-406.

[4] S. Nouraei, S. Middleton, C. Butler, G. Sandhu, An estimation of the population incidence of adult unilateral vocal fold mobility impairment in England, Logoped Phoniatr Vocol 40 (2015) 93-94.

[5] C. Walton, P. Carding, K. Flanagan, Perspectives on voice treatment for unilateral vocal fold paralysis, Curr Opin Otolaryngol Head Neck Surg 3 (2018) 157-161.

[6] C. Walton, P. Carding, E. Conway, K. Flanagan, H. Blackshaw, Voice outcome measures for adult patients with unilateral vocal fold paralysis: A systematic review, The Laryngoscope 129 (2018) 187-197. 
[7] J.C. Lucero, A. Van Hirtum, N. Ruty, J. Cisonni, X. Pelorson., Validation of theoretical models of phonation threshold pressure with data from a vocal fold mechanical replica, J Acoust Soc Am 125 (2009) 632-635.

[8] A. Van Hirtum, J. Cisonni, N. Ruty, X. Pelorson., I. Lopez, F. van Uittert, Experimental validation of some issues in lip and vocal folds physical models, Acta Acustica 93 (2007), 314-323.

[9] N. Ruty, X. Pelorson., A. Van Hirtum, I. Lopez, A. Hirschberg, An in-vitro setup to test the relevance and the accuracy of low-order models of the vocal folds, J Acoust Soc Am 121 (2007) 479-490.

[10] J.C. Lucero, X. Pelorson., A. Van Hirtum, Phonation threshold pressure at large asymmetries of the vocal folds, Biomed Signal Process Control 62 (2020) 102105.

[11] P. Murray, S. Thomson, Vibratory responses of synthetic, self-oscillating vocal fold models, J Acous Soc Am 132 (2012) 3428-3438.

[12] A. Bouvet, I. Tokuda, X. Pelorson, A. Van Hirtum, Influence of level difference due to vocal folds angular asymmetry on auto-oscillating replicas, J. Acoust. Soc. 147 (2020) 1136-1145.

[13] A. Bouvet, I. Tokuda, X. Pelorson, A. Van Hirtum, Imaging of autooscillating vocal folds replicas with left-right level difference due to angular asymmetry, Biomed Signal Process Control 63 (2021) 1-12.

[14] J. G. Svec, On vibration properties of human vocal folds, Ph.D. thesis, University of Groningen (2000).

[15] P. Mergell, H. Herzel, I.R. Titze, Irregular vocal-fold vibration - High-speed observation and modeling, J Acous Soc Am 108 (2000) 2996-3002.

[16] Z. Zhang, J. Neubauer, A. Berry, Aerodynamically and acoustically driven modes of vibration in a physical model of the vocal folds, J Acous Soc Am 120 (2006) 2841-2849. 
[17] Z. Zhang, J. Neubauer, A. Berry, Influence of vocal fold stiffness and acoustic loading on flow-induced vibration of a single-layer vocal fold model, J Sound Vib 322 (2009) 299-313.

[18] Z. Zhang, The influence of material anisotropy on vibration at onset in a three-dimensional vocal fold model, J Acous Soc Am 135 (2014) 1480-1490.

[19] D.A. Berry, H. Herzel, I.R. Titze, K. Krischer, Interpretation of biomechanical simulations of normal and chaotic vocal fold oscillations with empirical eigenfunctions, J Acous Soc Am 95 (1994) 3595-3604.

[20] R. Scherer, D. Shinwari, K. De Witt, C. Zhang, B. Kucinschi, A. Afjeh, Intraglottal pressure profiles for a symmetric and oblique glottis with a divergence angle of 10 degrees, J Acous Soc Am 109 (2001) 1616-1630.

[21] I. Tokuda, R. Shimamura, Effect of level difference between left and right vocal folds on phonation: Physical experiment and theoretical study, J Acous Soc Am 142 (2017) 482-492.

[22] B. Pickup, S. Thomson, Flow-induced vibratory response of idealized versus magnetic resonance imaging-based synthetic vocal fold models, J Acous Soc Am 128 (2010) 124-129.

[23] J. Tu, R. C.W., D. Luchtenburg, S. Brunton, J. Kutz, On dynamic mode decomposition: Theory and applications, J Comp Dynamics 1 (2014) 391465 421.

[24] J. Kutz, S. Brunton, B. Brunton, J. Proctor, Dynamic mode decomposition: data-driven modeling of complex systems, SIAM, Philadelphia, USA, 2016.

[25] W. Boyce, R. DiPrima, Elementary Differential Equations, Whiley, 2008. 